\title{
EXTRAÇÃO E PURIFICAÇÃO DE FÁRMACOS ANTI-INFLAMATÓRIOS NÃO ESTEROIDAIS CICLO- OXIGENASE-2 SELETIVOS
}

\author{
Tailane Sant'Anna Moreira, Yara Peluso Cid, Maria Bernadete Riemma Pierre e Valeria Pereira de Sousa* \\ Departamento de Medicamentos, Faculdade de Farmácia, Universidade Federal do Rio de Janeiro, 21941-902 Rio de Janeiro - RJ, \\ Brasil \\ Arthur Eugen Kummerle e Carlos Alberto Manssour Fraga \\ Departamento de Fármacos, Faculdade de Farmácia, Universidade Federal do Rio de Janeiro, 21941-902 Rio de Janeiro - RJ, \\ Brasil
}

Recebido em 19/6/08; aceito em 13/1/09; publicado na web em 30/4/09

\begin{abstract}
EXTRACTION AND PURIFICATION OF SELECTIVES CICLOOXIGENASE-2 NONSTEROIDAL ANTIINFLAMMATORY DRUGS. Celecoxib (CB) and lumiracoxib (LM) are potent COX-2 inhibitors widely marketed for the treatment of rheumatoid arthritis and osteoarthritis. Nevertheless, it is difficult to obtain because it are protected under patents. The aim of this work was to develop an extraction method of drugs, CB and LM, in order to obtain the drug with a purity degree appropriated for use in research projects. The developed method showed to be effective of both drugs, becoming interesting due to its low cost, easy and speed of execution, application to different dosage forms (capsules and tablets) and drugs with different physicochemical properties.
\end{abstract}

Keywords: celecoxib; lumiracoxib; extraction.

\section{INTRODUÇÃO}

Os anti-inflamatórios não esteroidais (AINEs) são fármacos amplamente utilizados no tratamento da dor, inflamação e febre. O mecanismo de ação dos compostos desta classe terapêutica envolve a inibição da enzima araquidonato ciclo-oxigenase (COX), a enzimachave para a síntese dos prostanóides e tromboxanos. Os prostanóides são mediadores celulares que modulam uma enorme variedade de processos fisiológicos e patológicos através de receptores de membrana localizados na superfície das células alvo. ${ }^{1}$ Estas sustâncias têm importante função homeostática na proteção da mucosa gástrica, fisiologia renal, gestação e agregação plaquetária, além de terem sua produção induzida em condições como inflamação e câncer. ${ }^{2}$ Desta forma, através da inibição da COX, os AINEs produzem os efeitos terapêuticos, mas também, numa maior ou menor extensão, alguns efeitos adversos como ulcerações, sangramentos, perfurações e obstruções gastrintestinais. , $^{3,4}$

Atualmente, sabe-se que existem duas isoformas da enzima ciclooxigenase: COX-1 e COX-2. A primeira é uma enzima constitutiva, expressa na maioria dos tecidos do organismo, agindo na produção de prostaglandinas que controlam os processos fisiológicos normais. ${ }^{5}$ A segunda é não constitutiva e tem sua expressão aumentada, principalmente, nos processos inflamatórios. ${ }^{6}$

Os primeiros AINES desenvolvidos (indometacina, naproxeno, ibuprofeno, entre outros) eram inibidores não seletivos das isoformas de COX e, apesar de terem eficácia comprovada quanto ao efeito antiinflamatório evidenciado, têm uso contínuo limitado devido a efeitos adversos gastrintestinais como displasia e dor abdominal, além de perfuração ou sangramento gastroduodenal em menor proporção. Esses efeitos adversos apresentados são oriundos da inibição da isoforma 1 da COX. Desse modo, a descoberta da segunda isoforma permitiu o desenvolvimento de uma subclasse de AINEs, os inibidores COX-2 seletivos, que apresentam o efeito terapêutico com a mesma eficácia, sem provocar os efeitos adversos indesejáveis oriundos da inibição da COX-1. Entre esses novos AINEs pode-se incluir celecoxibe,

\footnotetext{
*e-mail: valeria@ pharma.ufrj.br
}

lumiracoxibe e etoricoxibe, indicados principalmente no tratamento da osteoartrite, artrite reumatóide, dor aguda e alívio dos sintomas de dismenorréia primária.,

O celecoxibe (CB), (4-[5-(4-metilfenil)-3-(trifluorometil)-1Hpirazol-1-il] benzenesulfonamida) (Figura 1), é um derivado da classe das aril-sulfonamidas que mostra características apolares sendo, portanto, um fármaco lipofílico e de alta permeabilidade, pertencente à classe biofarmacêutica II. Apresenta características de um ácido fraco com valores de pka 11,1, portanto, encontra-se na forma não ionizada em $\mathrm{pH}$ fisiológico. ${ }^{8}$

O lumiracoxibe (LM), quimicamente conhecido como ácido 2-[(2-fluor-6-clorofenil)amino]-5-metil-benzenoacético (Figura 1), é o mais recente AINE COX-2 seletivo desenvolvido e quimicamente diferente dos outros coxibes, por não possuir o grupamento farmacofórico sulfonamida ou sulfona e sim um ácido carboxílico em sua estrutura, que confere à molécula propriedades ácidas fracas (pKa 4,7). Além disso, LM apresenta Log P 1,15 como reflexo da presença de grupos com características lipofílicas. ${ }^{9,10}$

Apesar de amplamente comercializados no mundo, os AINEs COX-2 seletivos são de difícil obtenção por estarem sob proteção patentária. Desta forma, o objetivo deste trabalho foi desenvolver um método de extração de fármacos com propriedades físico-químicas distintas, celecoxibe (CB) e lumiracoxibe (LM), a partir de formas farmacêuticas sólidas de maneira a se obter o fármaco com pureza desejada para utilização em projetos de pesquisa no âmbito do desenvolvimento de novas formulações farmacêuticas.

\section{PARTE EXPERIMENTAL}

\section{Reagentes e material}

Os fármacos CB e LM foram extraídos e purificados a partir de medicamentos comprados em farmácias locais. OCB foi obtido a partir do medicamento Celebra ${ }^{\circledR}$ (Pfizer Pharmaceuticals LLC, Caguas, Porto Rico), na forma de cápsula oral de $200 \mathrm{mg}$ lote BE0002. Foi utilizado padrão de trabalho com teor 99,4\% (lote: CXB-02/07-08) de CB (Exim-Pharm International, Mumbai, Índia). O LM foi obtido a partir do medicamento 


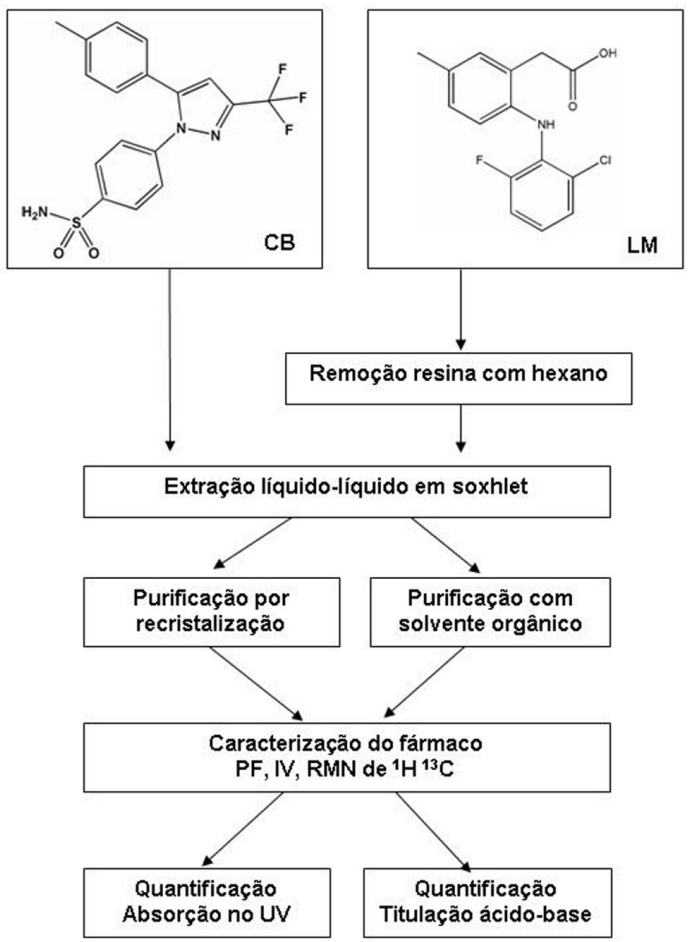

Figura 1. Esquema representativo do processo de extração, purificação, caracterização e quantificação do CB e LM

Prexige ${ }^{\circledR}$ (Novartis Biociências S.A., Stein, Suíça), comercializado na forma de comprimido oral de $400 \mathrm{mg}$ (lote: Z20032).

Foram utilizados os reagentes de grau analítico: hidróxido de sódio $(\mathrm{NaOH})$, ácido clorídrico $(\mathrm{HCl})$, fosfato de sódio, acetonitrila, acetato de etila, etanol, cloreto de sódio $(\mathrm{NaCl})$ da Vetec (Rio de Janeiro, Brasil). O brometo de potássio ( $\mathrm{KBr}$ ) e biftalato de potássio foram obtidos da Sigma-Aldrich Co. (Montana, EUA). A água purificada foi obtida usando sistema de purificação Milli-Q da Millipore Co. (Massachusetts, USA).

\section{Procedimento de extração}

O processo de extração do LM a partir dos comprimidos revestidos foi iniciado com uma etapa prévia para eliminar a resina de revestimento. Procedeu-se à adição de hexano a uma massa relativa a 6 comprimidos, correspondente a 2,4 g do fármaco, previamente triturados em gral de porcelana. A mistura foi mantida sob agitação por 30 min e, então, filtrada com papel de filtro. O resíduo sólido foi seco no dessecador por 24 h. O CB foi obtido da extração realizada diretamente a partir do conteúdo de 30 cápsulas, correspondente a 6 g do fármaco, sem a etapa prévia à extração.

O resíduo sólido do $\mathrm{LM}$ e o conteúdo das cápsulas de $\mathrm{CB}$ foram extraídos em $300 \mathrm{~mL}$ de água contida em balão de fundo redondo com auxílio de um sohxlet em refluxo por $2 \mathrm{~h}$. A fração aquosa foi descartada e o resíduo sólido foi, sequencialmente, extraído em 300 $\mathrm{mL}$ de etanol contido em balão de fundo redondo por cerca de $4 \mathrm{~h}$. A solução etanólica obtida foi evaporada em rotavapor a $100 \mathrm{rpm}$ em banho termostatizado à $\pm 70{ }^{\circ} \mathrm{C}$, levando à obtenção de um resíduo sólido bruto que foi objeto de subsequente purificação.

\section{Purificação do resíduo de extração}

Cada 5 g do resíduo sólido bruto de LM foi purificado após dissolução em $400 \mathrm{~mL}$ de solução aquosa de $\mathrm{NaOH} 0,1 \mathrm{M}$, seguida de filtração da mistura em papel de filtro. O filtrado obtido foi acidificado com $\mathrm{HCl} 10 \%$ até $\mathrm{pH}$ 4,0 e após a precipitação foi adicionado 200 $\mathrm{mL}$ de solução saturada de $\mathrm{NaCl}$. A solução resultante foi extraída com $200 \mathrm{~mL}$ de acetato de etila. Após toda a extração, a fase orgânica obtida foi lavada duas vezes com $100 \mathrm{~mL} \mathrm{NaOH} \mathrm{0,1} \mathrm{M.} \mathrm{O} \mathrm{LM} \mathrm{obtido}$ na fase aquosa foi precipitado pela adição de $\mathrm{HCl} 10 \%$ até $\mathrm{pH} 4,0$ em banho de gelo.

O resíduo sólido bruto obtido na extração do $\mathrm{CB}$ foi purificado através de recristalização com solvente. Aqueceu-se concomitantemente etanol e água destilada até ebulição em recipientes distintos. Adicionou-se etanol ainda quente ao resíduo sólido de CB contido em béquer sob agitação até completa solubilização, adicionou-se então a água até o aparecimento de turvação. A solução foi resfriada até a completa precipitação do fármaco.

Os precipitados formados foram filtrados em papel de filtro e secos por $24 \mathrm{~h}$ em dessecador até peso constante.

\section{Caracterização dos fármacos}

Para caracterização dos fármacos CB e LM extraídos e purificados foram realizados ensaios de faixa de fusão, espectrometria no infravermelho e ressonância magnética nuclear de carbono e hidrogênio.

Para a determinação da faixa de fusão foi utilizado o método, ${ }^{11}$ onde as amostras em triplicata foram introduzidas em capilares de vidro e levadas ao equipamento de determinação de ponto de fusão (Büchi, Flawil, Suíça).

Para a obtenção dos espectros no infravermelho, foram preparadas pastilhas de $\mathrm{KBr}$ contendo $3 \%$ (p/p) do fármaco, as quais foram levadas ao espectrofotômetro de infravermelho (Shimadzu, Kyoto, Japão), realizando-se varredura do espectro entre 400 e $4000 \mathrm{~cm}^{-1}$. As bandas observadas foram caracterizadas de acordo com os grupos funcionais que as representam.

Os espectros de ressonância magnética nuclear ( $\mathrm{RMN} \mathrm{de} \mathrm{H}^{1} \mathrm{e} \mathrm{C}^{13}$ ) foram obtidos à 200 e $50 \mathrm{MHZ}$, respectivamente, em espectrômetro (Bruker, Madison, USA), após dissolução das amostras de CB e LM dimetilsulfóxido deuterado (DMSO- $d_{6}$ ).

\section{Quantificação dos fármacos obtidos}

O doseamento de LM obtido foi realizado através de método analítico desenvolvido pelo próprio grupo, ${ }^{12}$ que se baseia na titulação de neutralização ácido-base com determinação potenciométrica do ponto de equivalência. Antes de cada análise, a solução titulante de $\mathrm{NaOH} 0,1 \mathrm{M}$ foi preparada e aferida com biftalato de potássio previamente dessecado por $4 \mathrm{~h}$ à $105^{\circ} \mathrm{C}$. Foram realizadas 5 determinações do LM pesando-se, analiticamente, cerca de $0,15 \mathrm{~g}$ de LM no copo do titulador automático, onde foram dissolvidos em $40 \mathrm{~mL}$ de etanol. As amostras foram levadas ao ultrassom por $5 \mathrm{~min}$ com agitação manual até a completa solubilização antes de se proceder à titulação. Utilizou-se a média e o desvio padrão relativo (DPR) de 5 determinações.

A determinação do teor de $\mathrm{CB}$ após extração e recristalização foi realizada por espectrofotometria de absorção no UV, conforme descrito por Saha e colaboradores, ${ }^{13}$ utilizando a média e o DPR de 3 determinações.

Para o preparo da solução estoque foi transferido, cerca de $20 \mathrm{mg}$ de CB padrão de trabalho $(99,4 \%)$ para um balão volumétrico de 200 $\mathrm{mL}$, cujo volume foi completado com diluente acetonitrila:tampão fosfato de sódio pH 5,6 (50:50), resultando em solução com concentração de $100 \mu \mathrm{g} / \mathrm{mL}$. A partir desta solução foram preparadas os 5 niveis de concentração para a obtenção da curva de calibração na faixa de 1,0 a $20,0 \mu \mathrm{g} / \mathrm{mL}$ de $\mathrm{CB}$. 
Uma quantidade equivalente a cerca de $20 \mathrm{mg}$ de $\mathrm{CB}$ purificado foi pesada e transferida para um balão de $200 \mathrm{~mL}$. Adicionou-se $150 \mathrm{~mL}$ de diluente e homogeneizou-se por $10 \mathrm{~min}$ no ultrassom. O volume foi completado com o diluente e foram realizadas diluições subseqüentes, a fim de se obter uma concentração final de $10 \mu \mathrm{g} / \mathrm{mL}$. As soluções foram preparadas em triplicata.

\section{RESULTADOS E DISCUSSÃO}

A Figura 1 apresenta o esquema do processo de extração, purificação, caracterização e quantificação do CB e LM.

O rendimento, em massa, do processo de extração e recristalização foi de $76,8 \%$ para o CB e 57,52\% para o LM.

A determinação do ponto de fusão foi realizada em triplicata. As faixas encontradas foram de 161 a $163{ }^{\circ} \mathrm{C}$, para o $\mathrm{CB}$, e de 156 a 158 ${ }^{\circ} \mathrm{C}$, para o LM. Esses valores estão próximos aos valores encontrados na literatura, a $157-159^{\circ} \mathrm{C}$, para ambos os fármacos CB e LM..$^{14,15}$

O uso da espectroscopia de infravermelho permitiu a identificação dos compostos através da análise de seus grupos funcionais. O LM apresentou uma banda alargada entre 3250 e $2500 \mathrm{~cm}^{-1}$, referente à deformação axial de ligações $\mathrm{O}-\mathrm{H}$, e outra intensa em $1672 \mathrm{~cm}$ ${ }^{1}$, característica de deformação axial de ligações $\mathrm{C}=\mathrm{O}$ de ácidos, indicando a presença do grupamento ácido fenilacético. A banda de deformação axial referente à amina secundária presente entre as duas fenilas deste composto também foi visualizada com deslocamento de $3358 \mathrm{~cm}^{-1}$. Apesar de apresentarem menor intensidade, também foi possível identificar a presença de bandas de combinação e de harmônicas de aromáticos em 1905 e $1844 \mathrm{~cm}^{-1}$. O CB pôde ser identificado principalmente pela presença de bandas bem intensas e características de deformações axiais de ligações $\mathrm{N}-\mathrm{H}$ e $\mathrm{S}=\mathrm{O}$ de sulfonamidas. A ligação N-H gerou duas bandas, características de sulfonamidas primárias, em 3340 e $3233 \mathrm{~cm}^{-1}$. Da mesma forma, a ligação $\mathrm{S}=\mathrm{O}$ originou duas bandas em 1348 e $1163 \mathrm{~cm}^{-1}$. Os aromáticos também puderam ser identificados pela presença de seis bandas: uma em $3098 \mathrm{~cm}^{-1}$ referente à deformação axial da ligação C-H de aromáticos, outras duas em 2919 e $2851 \mathrm{~cm}^{-1}$, geradas pela deformação axial da ligação C-H da metila ligada ao anel aromático, e as últimas três em 1914, 1801 e $1677 \mathrm{~cm}^{-1}$ referentes às bandas de combinação e de harmônicas de aromáticos.

As atribuições de deslocamento químico descritas na Tabela 1, obtidas através da análise dos espectros de RMN de carbono e hidrogênio do CB (Figura 2) e LM (Figura 3), estão de acordo com a literatura confirmando a identificação dos fármacos estudados, evidenciando a ausência de sustâncias contaminantes, comprovando a pureza do fármaco obtido por extração e demonstrando a eficácia do processo de obtenção utilizado. ${ }^{16,17}$

Após extração e purificação, CB e LM foram quantificados por titulação de neutralização ácido-base e por espectrofotometria de UV-Vis, respectivamente. O teor médio e o DPR obtidos para CB e LM foram $96,6 \pm 0,4 \%$ e $97,9 \pm 0,08 \%$, respectivamente, mostrandose adequados para o emprego no desenvolvimento de novas formas farmacêuticas para estes fármacos.

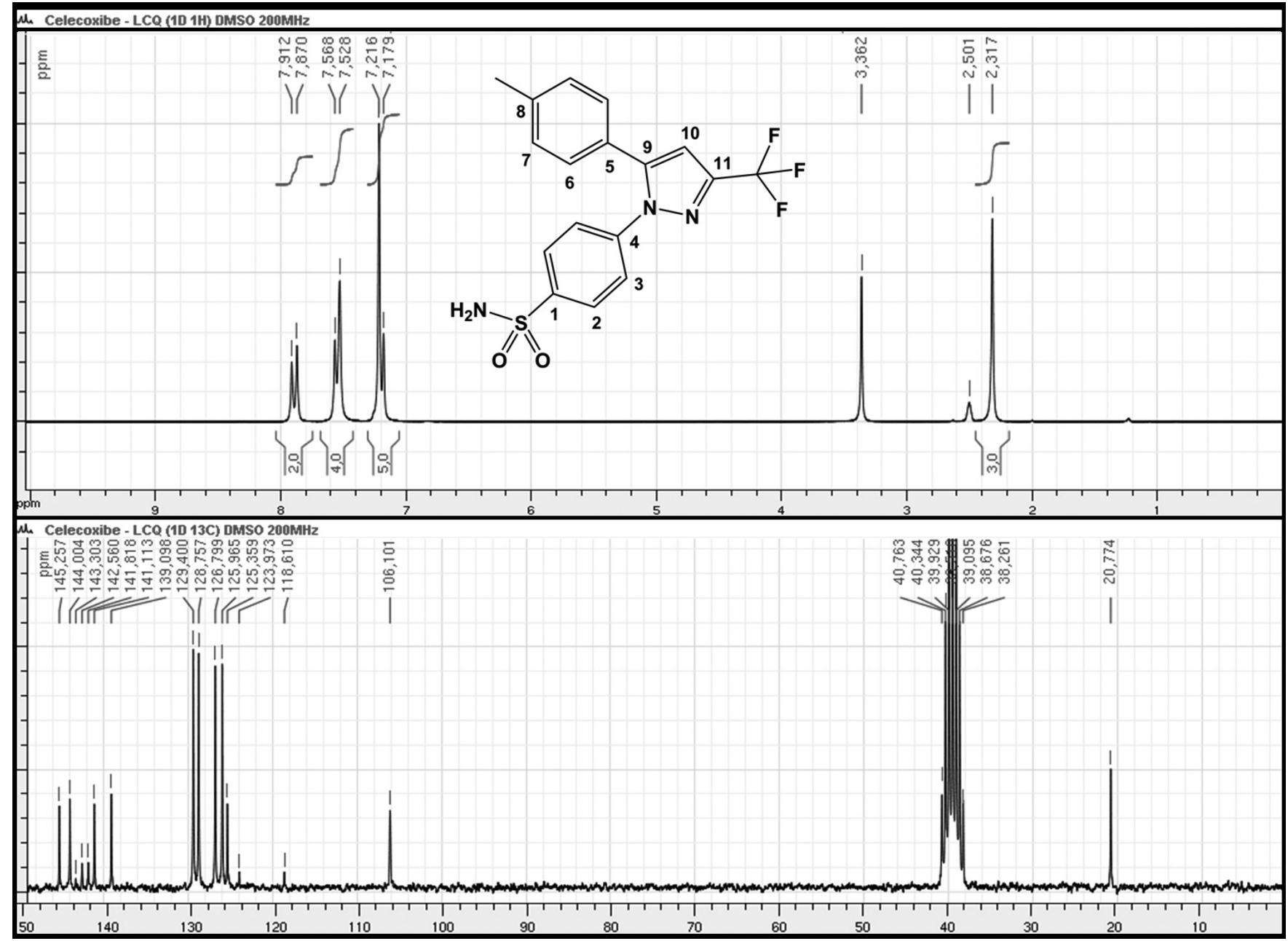

Figura 2. Espectro de RMN de ${ }^{1} \mathrm{H}$ e $\mathrm{RMN}$ de ${ }^{13} \mathrm{C}$ do $\mathrm{CB}$ 


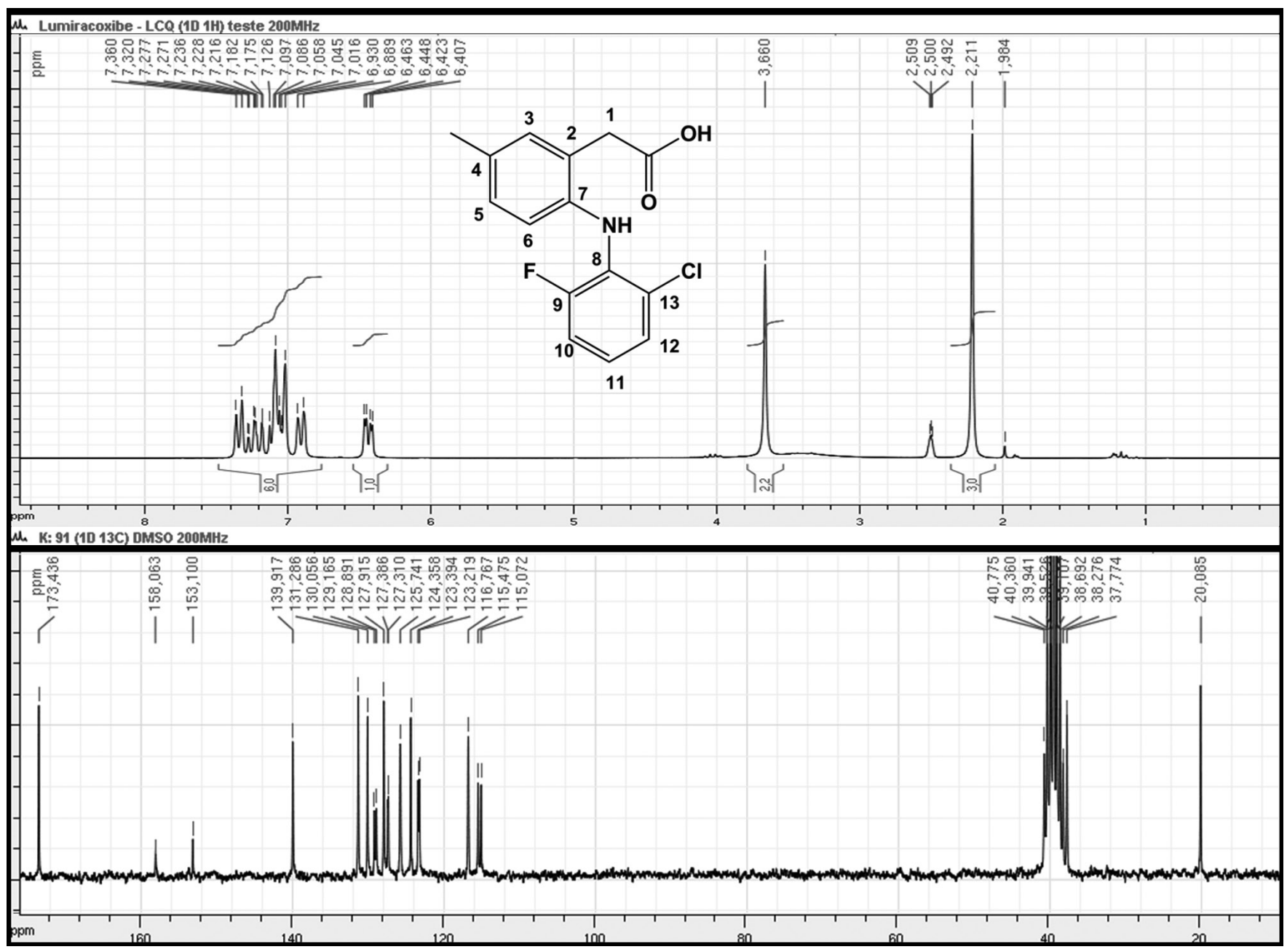

Figura 3. Espectro de RMN de ${ }^{1} \mathrm{H}$ e $R M N$ de ${ }^{13} \mathrm{C}$ do $\mathrm{LM}$

Tabela 1. Dados espectroscópicos de RMN de ${ }^{1} \mathrm{H}$ e RMN de ${ }^{13} \mathrm{C}(200$ e $50 \mathrm{MHz}$ ) do $\mathrm{CB}$ e LB

\begin{tabular}{|c|c|c|}
\hline & Celecoxibe & Lumiracoxibe \\
\hline $\begin{array}{l}{ }^{1} \mathrm{H} \text { RMN } \\
\left(\mathrm{DMSO}-d_{6}\right)\end{array}$ & $\begin{array}{c}\delta 2,32(\mathrm{~s}, 3 \mathrm{H}) ; 7,19(\mathrm{~d}, 5 \mathrm{H}, \\
\left.\mathrm{H}_{7}, \mathrm{H}_{10},-\mathrm{NH}_{2}\right) ; 7,54(\mathrm{~d}, \\
\left.4 \mathrm{H}, \mathrm{H}_{2}, \mathrm{H}_{6}\right) ; 7,89(\mathrm{~d}, 2 \mathrm{H}, \\
\left.\mathrm{H}_{3}, J=8,3 \mathrm{~Hz}\right)\end{array}$ & $\begin{array}{c}\delta 2,21\left(\mathrm{~s}, 3 \mathrm{H},-\mathrm{CH}_{3}\right) ; 3,66 \\
\left(\mathrm{~s}, 2 \mathrm{H},-\mathrm{CH}_{2}-\right) ; 6,43(\mathrm{dd}, \\
\left.\mathrm{H}_{6}, J=8,0 \mathrm{~Hz}, J_{H-F}=3,0 \mathrm{~Hz}\right) ; \\
6,90\left(\mathrm{~d}, \mathrm{H}_{5}, J=8.0 \mathrm{~Hz}\right) ; \\
7,02-7,13\left(\mathrm{~m}, 3 \mathrm{H}, \mathrm{H}_{3},\right. \\
\left.\mathrm{H}_{11},-\mathrm{NH}-\right) ; 7,20(\mathrm{ddd}, \\
\mathrm{H}_{10}, J=8,5 \mathrm{~Hz}, 1,5 \mathrm{~Hz}, \\
\left.J_{H-F}=11 \mathrm{~Hz}\right) ; 7,34\left(\mathrm{~d}, \mathrm{H}_{12},\right. \\
J=8.5 \mathrm{~Hz})\end{array}$ \\
\hline $\begin{array}{l}{ }^{13} \mathrm{C} \text { RMN } \\
\left(\mathrm{DMSO}-d_{6}\right)\end{array}$ & $\begin{array}{c}\delta 20,8\left(-\mathrm{CH}_{3}\right) ; 106,1\left(\mathrm{C}_{10}\right) \\
118,6 \text { e } 124,0\left(-\mathrm{CF}_{3}\right) ; \\
125,4\left(\mathrm{C}_{5}\right) ; 126,0\left(\mathrm{C}_{3}\right) \\
126,8\left(\mathrm{C}_{6}\right) ; 128,8\left(\mathrm{C}_{2}\right) \\
129,4\left(\mathrm{C}_{7}\right) ; 139,1\left(\mathrm{C}_{1}\right) ; \\
141,1(\mathrm{C}) ; 141,1,141,8 \\
142,6,143,3\left(\mathrm{C}_{11}\right) ; 144,0 \\
\left(\mathrm{C}_{4}\right) ; 145,3\left(\mathrm{C}_{9}\right)\end{array}$ & $\begin{array}{c}\delta 20,1\left(-\mathrm{CH}_{3}\right) ; 37,8 \\
\left(-\mathrm{CH}_{2}-\right) ; 115.3\left(\mathrm{~d}, \mathrm{H}_{10},\right. \\
\left.J_{C-F}=20 \mathrm{~Hz}\right) ; 116,8\left(\mathrm{C}_{6}\right) ; \\
123,3\left(\mathrm{~d}, \mathrm{C}_{11}, J_{C-F}=9,0 \mathrm{~Hz}\right) ; \\
124,4\left(\mathrm{C}_{2}\right) ; 125,7\left(\mathrm{C}_{12}\right) ; \\
127,4\left(\mathrm{~d}, \mathrm{C}_{13}, J_{C-F}=4,0 \mathrm{~Hz}\right) ; \\
127,9\left(\mathrm{C}_{5}\right) ; 129,0\left(\mathrm{~d}, \mathrm{C}_{8},\right. \\
\left.J_{C-F}=14,0 \mathrm{~Hz}\right) ; 130,1\left(\mathrm{C}_{4}\right) ; \\
131,3\left(\mathrm{C}_{3}\right) ; 140,0\left(\mathrm{C}_{7}\right) ; \\
155,5\left(\mathrm{~d}, \mathrm{C}_{9}, J_{C-F}=247 \mathrm{~Hz}\right) ; \\
173,4\left(\mathrm{CO}_{2} \mathrm{H}\right)\end{array}$ \\
\hline
\end{tabular}

\section{CONCLUSÕES}

O método desenvolvido para a extração de anti-inflamatórios COX2 seletivos a partir de formas farmacêuticas mostrou-se eficaz para a obtenção de ambos os fármacos, CB e LM, com pureza desejada. O método torna-se vantajoso por ser aplicável para formas farmacêuticas diferentes e para fármacos com propriedades físico-químicas distintas. É um método simples, de baixo custo e eficaz, sendo uma ótima alternativa para a obtenção de fármacos de difícil aquisição comercial.

\section{AGRADECIMENTOS}

À CAPES e FAPERJ pelas bolsas e apoios concedidos.

\section{REFERÊNCIAS}

1. Fitzpatrick, F. A.; Soberman, R.; J. Clin. Invest. 2001, 107, 1347.

2. Fitzgerald, G. A.; Am. J. Cardiol. 2002, 89, 26 D.

3. Bombardier, C.; Laine, L.; Reicin, A.; New Engl. J. Med. 2000, 343, 1520

4. Fitzgerald, G. A.; Patrono, C. P.; New Engl. J. Med. 2001, 345, 433.

5. Lages, A. S.; Romeiro, N. C.; Fraga, C. A. M.; Barreiro, E. J.; Quim. Nova 1998, 21, 761.

6. Smith, W. L.; Dewitt, D. L.; Garavito, R. M.; Annu. Rev. Biochem. 2000, 69,145 .

7. Silverstein, F. E.; Faich, G.; Goldstein, J. L.; JAMA 2000, 284, 1247. 
8. Paulson, S.; Vaughn, M.; Jessen, S.; Lawal, Y.; Gresk, C.; Yan, B.; Maziasz, T.; Cook, C.; Karim, A.; J. Pharmacol. Exp. Ther. 2001, 297, 638.

9. Guensens, P.; Alten, R.; Rovensky, J.; Sloan V. S.; Krammer, G.; Kralidis, G.; Richardson, P.; Int. J. Clin. Pract. 2004, 58, 1033.

10. Friecke, J.; Davis, N. Y. U.; Krammer, G.; J. Pain 2008, 9, 20.

11. Farmacopéia Brasileira; $4^{\mathrm{a}}$ ed., Atheneu: São Paulo, 1988.

12. Moreira, T. M.; Desenvolvimento de Sistemas de Liberação Transdérmicos para o Antiinflamatório Lumiracoxibe: Obtenção, Avaliação Físico-Química e Estudos de Permeação in vitro em Pele de Modelo Animal, Universidade Federal do Rio de Janeiro, Brasil, 2008.

13. Saha, R. N.; Sajeev, C.; Jadhav, P. R.; Patil, S. P.; Srinivasan, N.; J. Pharm. Biomed. Anal. 2002, 28, 741.
14. The Merck Index; An Encyclopedia of Chemicals, Drugs, and Biologicals, $13^{\text {th }}$ ed., Merck Research Laboratories, Whitehouse Station, 2001

15. Fugimoto, R. A.; Mugrare, B.; Mcquire, L. W.; Duzer, J. H. V.; WO 99/11605, 1999.

16. Penning, T. D.; Talley, J. J.; Bertenshaw, S. R.; Carter, J. S.; Collins, P. W.; Docter, S.; Graneto, M. J.; Lee, L. F.; Malecha, J. W.; Miyashiro, J. M.; Rogers, R. S.; Rogier, D. J.; Yu, S. S.; Anderson, G. D.; Burton, E. G.; Cogburn, J. N.; Gregory, S. A.; Koboldt, C. M.; Perkins, W. E.; Seibert, K.; Veenhuizen, A. W.; Zhang, Y. Y.; Isakson, P. C.; J. Med. Chem. 1997, 40, 1347.

17. Acemoglu, M.; Allmendinger, T.; Calienni, J.; Cercus, J.; Loiseleur, O.; Sedelmeier, G. H.; Xu, D.; Tetrahedron 2004, 60, 11571. 